\title{
Tenofovir containing thiolated chitosan core/shell nanofibers: in vitro and in vivo evaluations
}

\author{
Jianing Meng ${ }^{a *}$, Vivek Agrahari ${ }^{a *}$, Miezan J. Ezoulin ${ }^{a *}$, Chi Zhang ${ }^{b}$, Sudhaunshu S. \\ Purohit $^{\text {b }}$, Agostino Molteni ${ }^{\mathrm{c}}$, Daniel Dim ${ }^{\mathrm{d}}$, Nathan A. Oyler ${ }^{\mathrm{b}}$, Bi-Botti C. Youan ${ }^{\mathrm{a}^{* \star}}$
}

\author{
${ }^{a}$ Laboratory of Future Nanomedicines and Theoretical Chronopharmaceutics Division \\ of Pharmaceutical Sciences, University of Missouri-Kansas City, Kansas City, Missouri \\ 64108, US \\ ${ }^{b}$ Department of Chemistry, University of Missouri-Kansas City, Kansas City, Missouri \\ 64110, US
}

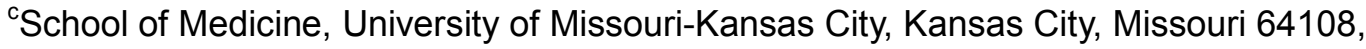
US

${ }^{\mathrm{d}}$ Truman Medical Center Hospital Hill, Kansas City, Missouri 64110, US

${ }^{*}$ Author contributed equally

** Correspondence to: Bi-Botti C. Youan , Tel: (1)816-235-2410; Fax: (1)816-235-5779;

Email: youanb@umkc.edu 


\section{Synthesis of chitosan-thioglycolic acid conjugates (TCS)}

Chitosan-thioglycolic acid conjugates (TCS) is synthesized using a previously described method [1]. Briefly, chitosan (100 mg) is dissolved in $\mathrm{HCl}(10 \mathrm{ml}, 0.1 \mathrm{M})$ to obtain a solution of chitosan hydrochloride $(1 \% \mathrm{w} / \mathrm{v})$. Then thioglycolic acid TGA (100 mg) is added. After TGA is completely dissolved in the chitosan hydrochloride solution, EDC (10 mg, $0.05 \mathrm{mmol})$ and NHS (15 mg, $0.13 \mathrm{mmol})$ are added. The pH is adjusted to 5 and the reaction mixture is incubated for $3 \mathrm{~h}$ at room temperature under constant stirring (Supplementary Figure S1).. Control sample without EDC and

NHS is also

prepared and analyzed.
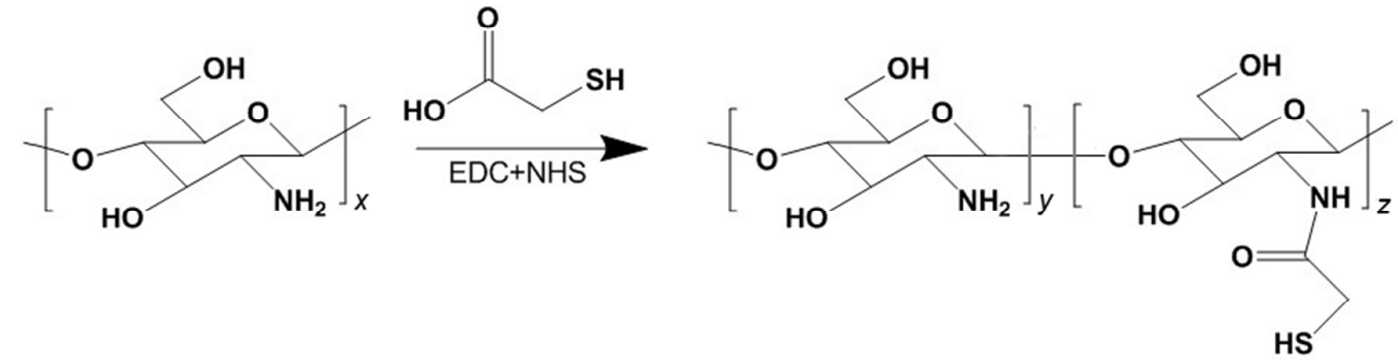

Supplementary Figure S2. Synthesis of chitosan-thioglycolic acid conjugates (TCS) using chitosan and thioglycolic acid.

The reaction mixture subsequently under goes dialysis for 3 days at $10^{\circ} \mathrm{C}$ in the dark against $\mathrm{HCl}(1 \mathrm{~L}, 5 \mathrm{mM})$ to isolate the pure chitosan conjugates and to eliminate unbound TGA, then against the same medium but containing $\mathrm{NaCl}(1 \%$ $\mathrm{w} / \mathrm{v}$ ) in order to reduce the ionic interactions between chitosan and the sulfhydryl compound.

TGA is covalently attached to chitosan by the formation of amide bonds between the carboxylic acid groups of the TGA and the primary amino groups of the chitosan. 
To achieve this, the carboxylic acid moieties of the TGA are activated by the EDC. The addition of NHS stabilizes the amine-reactive intermediate and increase the efficiency of the EDC-mediated coupling reactions. The synthesis is performed at a $\mathrm{pH}<5$ to avoid the formation of the disulfide bonds by air oxidation since, at $\mathrm{pH}<5$, the formation of disulfide bonds can be excluded due to the low concentration of the thiolate anions [1].

\section{Proton nuclear magnetic resonance ( $\left.{ }^{1} \mathrm{H}-\mathrm{NMR}\right)$ spectroscopy analysis of chitosan-TGA conjugates}

In order to confirm the effective conjugation between CS and TGA, proton $\left({ }^{1} \mathrm{H}\right)$ NMR spectroscopy analysis is performed. The samples are dissolved in deuterium oxide $\left(D_{2} \mathrm{O}\right)$. The spectra are observed on a Varian (Palo Alto, CA, USA) $400 \mathrm{MHz}$ spectrometer with a Varian two channel probe. $V_{\text {nmrj }} 2.2$ (Palo Alto, CA, USA) is used to process the experimental data. Typically, the spectra are acquired with relaxation delay $(2 \mathrm{sec})$ and the number of scans is 2048 . The concentration of each $500 \mu \mathrm{l}$ sample is $4 \mathrm{mg} / \mathrm{ml}$.

Based on the results of the NMR spectroscopy (Supplementary Figure S2), amide bond with chemical shift $\delta=8.22$ is indeed formed and identified in chitosan-TGA conjugate when EDC and NHS are used. The control, which is prepared by the same method as of the conjugates but omitting EDC and NHS, exhibits no peak of the amide bond. 


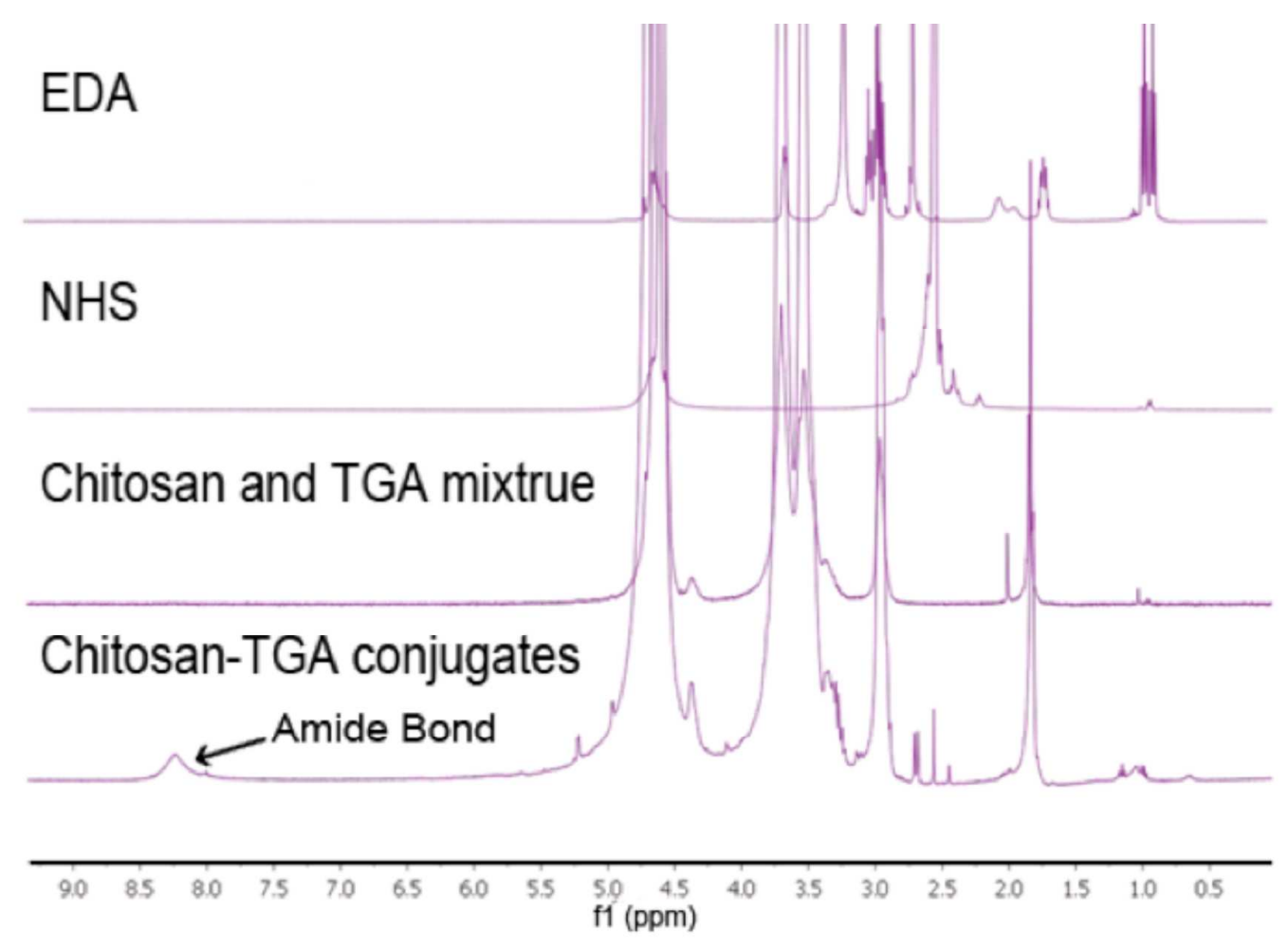

Supplementary Figure S2. ${ }^{1} \mathrm{H}$ NMR spectrum of the synthesized chitosan-TGA conjugate and starting materials.

\section{Morphological and surface chemical analysis}

For SEM analysis, small amount of NFs is mounted to aluminum stubs with conductive tape, and sputter coated with approximately $20 \mathrm{~nm}$ thickness of goldpalladium alloy. The NFs are then examined using a FEI/Philips XL30 FieldEmission Environmental SEM (Philips, Eindhoven, Netherlands) at $5 \mathrm{kV}$. Digital images are acquired with ORIUSTM SC 100011 Megapixel CCD camera (Gatan, Pleasanton, CA, USA). The SEM images are analyzed using Image Pro ${ }^{\circledR}$ Plus software (Image Pro Plus 6.0, Media Cybernetics, Silver Spring, MD, USA). The following measurements are made: average fiber diameter, average bead diameter, and the approximate ratio of bead area to total bead and fiber area. 
For TEM analysis, NFs are deposited onto a copper grid with a carbon support film. The copper grid is submerged in water and then dried. The NFs are observed under a Scanning Transmission Electron Microscope CM12 (FEl, Hillsboro, OR, USA) at $80 \mathrm{kV}$ accelerating voltage. Digital images are acquired as described above.

For FT-IR spectroscopy analysis, the NFs are directly scanned over the wavenumber range of 650 and $4000 \mathrm{~cm}^{-1}$ using a Nicolet ${ }^{\mathrm{TM}}$ iS ${ }^{\mathrm{TM}} 10 \mathrm{FT}-\mathrm{IR}$ Spectrometer (Thermo Scientific, Lenexa, KS, USA). The background spectra are collected at ambient conditions before analyzing each sample. The FT-IR spectra are recorded by OMNIC V 7.0 spectra software (Thermo Scientific, Lenexa, KS, USA).

\section{Cellular membrane integrity assay}

The NFs membrane with an area of $0.25 \mathrm{~cm}^{2}$ (approximately $0.1 \mathrm{mg}$ ), is peeled from the collector and laid at the bottom of each well in the 96-well plates. VK2/E6E7 and End1/E6E7 cell lines are seeded in the plates with the NF membrane to ensure $1 \times 10^{4}$ cells per well. The plates are then incubated at $37^{\circ} \mathrm{C}$ for 24 or $48 \mathrm{~h}$. The cells incubated with media alone or $1 \%$ Triton $\mathrm{X}-100$ (both without NFs) are used as the negative and positive controls, respectively.

To measure the lactate dehydrogenase (LDH) release, after the 24 or $48 \mathrm{~h}$ incubation time, the plates are taken out of the incubator and equilibrated to $22^{\circ} \mathrm{C}$. In each well, $100 \mu \mathrm{l}$ of CytoTox-ONE ${ }^{\mathrm{TM}}$ Reagent is added. The plates are shaken 
for $30 \mathrm{~s}$ and then incubated at $22{ }^{\circ} \mathrm{C}$ for $10 \mathrm{~min}$. After this, $50 \mu \mathrm{l}$ of stop solution is added and the fluorescence intensity is determined by a microplate reader at $\lambda_{\mathrm{ex}}$ $560 \mathrm{~nm}$ and $\lambda_{\mathrm{em}} 590$ [2].

\section{Cell viability assay}

To determine the cell viability, after the incubation time, the media in the wells is removed and substituted with fresh media. Twenty microliters of Cell Titer $96{ }^{\circledR}$ Aqueous One Solution Reagent (Promega, Madison, WI, USA) is added to each well and incubated at $37^{\circ} \mathrm{C}$ for $4 \mathrm{~h}$. The absorbance is recorded at a wavelength of $490 \mathrm{~nm}$.

\section{Lactobacillus viability assay}

The lactobacillus viability assay is performed using an established method [3].

The $L$. crispatus bacteria density is adjusted to an $\mathrm{OD}_{670}$ of 0.06 , corresponding to a $0.5 \mathrm{McF}$ arland Standard or $10^{8} \mathrm{CFU} / \mathrm{ml}[4]$. It is then plated in the plates with NFs at a volume of $100 \mu \mathrm{l}$ per well, and then incubated at $37^{\circ} \mathrm{C}$. The lactobacillus incubated without NFs is used as the negative control and those incubated with a commercially available $10 \mu \mathrm{g} / \mathrm{ml}$ of Penicillin-Streptomycin solution (Invitrogen, Carlsbad, CA, USA) is used as a positive control. After 24 or $48 \mathrm{~h}, 20 \mu \mathrm{l}$ of MTS reagent is added to each well, and the bacterial viability is determined by measuring the absorbance at a wavelength of $490 \mathrm{~nm}$ by a microplate reader.

\section{Hematoxylin and eosin (H\&E) staining}

Serial sections are stained with hemotoxylin and eosin (H\&E). Briefly, slides containing paraffin sections are deparaffinized in Xylene. To rehydrate the section, 
the slides are passed through several changes of alcohol and then rinsed thoroughly with deionized water. Hematoxylin is applied on the sections, over-staining is removed by rinsing in water. Then the slides are stained with eosin followed by washing again with water. In a typical tissue, Hematoxylin stains nucleic acids and has a deep blue color, Eosin stains proteins nonspecifically and has a pink staining. Therefore, nuclei are stained blue, whereas the cytoplasm and extracellular matrix are stained pink. Images are captured using a Nikon Labophot-2 microscope (Nikon Instruments, Inc., Melville, NY) equipped with a PAXCam digital microscope camera and analyzed using PAX-it image management and analysis software (Midwest Information Systems, Inc., Villa Park, Illinois).

\section{Immunohistochemical staining}

To identify the inflammatory cell (CD45) infiltrate, immunohistochemical staining is performed in genital tissue sections [5]. Briefly, the de-paraffinized and rehydrated tissue sections are pretreated with citrate buffer/0.05\%Tween-20 (antigen retrieval reagents) for 20 min using steam heat method, rinsed with Tris buffered saline/0.05\%Tween-20 three times, incubated with $3 \% \mathrm{v} / \mathrm{v}$ hydrogen peroxide in PBS for 10 min, and blocked with 10\% normal goat serum (Vector Laboratories, Burlingame, CA) for $2 \mathrm{~h}$. The slides are then incubated with the primary anti-CD45 antibody overnight in a humidified chamber at $4{ }^{\circ} \mathrm{C}$, rinsed three times with PBS, followed by application of biotinylated secondary antibody at room temperature for $1 \mathrm{~h}$. The cells are visualized using the DAB: Peroxidase Substrate 
Kit under a Nikon Labophot-2 microscope. The tissue is then counter-stained by hematoxylin (Sigma, Saint Louis, MO) and treated with alcohol gradient and xylene before application of a coverslip mounted using cytoseal 60 mounting media (Richard Allan Scientific, Kalamazoo, MI). Images are viewed and captured as described above. 


\section{REFERENCES}

[1] C.E. Kast, A. Bernkop-Schnurch, Thiolated polymers--thiomers: development and in vitro evaluation of chitosan-thioglycolic acid conjugates, Biomaterials 22(17) (2001) 2345-52.

[2] J. Meng, T. Zhang, V. Agrahari, M.J. Ezoulin, B.B. Youan, Comparative biophysical properties of tenofovir-loaded, thiolated and nonthiolated chitosan nanoparticles intended for HIV prevention, Nanomedicine 9(11) (2014) 1595-612.

[3] C. Lackman-Smith, C. Osterling, K. Luckenbaugh, M. Mankowski, B. Snyder, G. Lewis, J. Paull, A. Profy, R.G. Ptak, R.W. Buckheit, Jr., K.M. Watson, J.E. Cummins, Jr., B.E. Sanders-Beer, Development of a comprehensive human immunodeficiency virus type 1 screening algorithm for discovery and preclinical testing of topical microbicides, Antimicrob Agents Chemother 52(5) (2008) 1768-81.

[4] S.J. Klebanoff, R.W. Coombs, Viricidal effect of Lactobacillus acidophilus on human immunodeficiency virus type 1: possible role in heterosexual transmission, J Exp Med 174(1) (1991) 289-92.

[5] B.J. Catalone, T.M. Kish-Catalone, L.R. Budgeon, E.B. Neely, M. Ferguson, F.C. Krebs, M.K. Howett, M. Labib, R. Rando, B. Wigdahl, Mouse model of cervicovaginal toxicity and inflammation for preclinical evaluation of topical vaginal microbicides, Antimicrobial agents and chemotherapy 48(5) (2004) 1837-47. 